\title{
Extortion as an Obstacle to Economic Growth: A Dynamic Game Analysis*
}

\author{
H. Dawid $\quad$ G. Feichtinger $\quad$ A. Novak ${ }^{\ddagger}$
}

\begin{abstract}
In this paper we use a differential game analysis to study the dynamic strategic interaction between a criminal gang extorting money from local shop-owners and the local police force. In particular, we are interested in characterizing which factors are important in determining whether the capital stock of local shop-owners keeps growing in spite of extortion or the criminal activity leads to a phase of stagnation of the local economy. A Markov perfect equilibrium of the game is characterized in order to address this question and several policy implications are derived to facilitate growth in regions affected by extortion.
\end{abstract}

JEL Classification: C73, D92, K42

Keywords: Extortion, Differential Games, Capital Accumulation

\section{Introduction}

Organized crime is a significant source of concern for policy makers in many industrialized and non-industrialized countries. One of the major problems related to the activities of gangs and organized crime syndicates is the extortion of money from local business owners which makes the running of businesses less profitable and attractive in infected areas. This hinders the growth of capital stocks in regions where fast capital growth would be of particular importance. Since most instances of extortion attempts are not reported the exact amounts extorted by criminal organizations are not known

\footnotetext{
${ }^{*}$ The authors would like to thank J. Caulkins and J. Worth and two anonymous referees for their helpful comments.

${ }^{\dagger}$ Department of Economics, University of Southern California

${ }^{\ddagger}$ Department of Operations Research and Systems Theory, Vienna University of Technology

${ }^{\S}$ Department of Statistics and Decision Support Systems, University of Vienna
} 
but it is obvious that extortion is a serious problem for the local business owners in affected regions ${ }^{1}$ and has a significant impact on the economic growth in these areas. However, considering different regions where considerable extortion activity is documented, it becomes clear that there exist huge differences in the economic development of affected areas. Whereas activities of criminal organizations in regions like Southern Italy or East Asia seem to allow for certain - maybe reduced - local capital accumulation and economic growth, there are several examples of areas in major U.S. cities where gang related extortion has led to complete stagnation and a sharp decreases of local businesses ${ }^{2}$. According to The Economist (1994) three quarter of private enterprises in Russia are forced to pay up to $20 \%$ of their earnings to criminal gangs which is seen as one of the major reasons for the absence of significant economic growth ${ }^{3}$. Of course these differences may be partly explained by regional differences in the overall economic development, but it seems that also certain characteristics of the criminal organization, the police force and the local population have an impact on whether extortion leads to stagnation or not. A clear understanding of the factors determining the impact of extortion activities would be very helpful to design public policies which not only aim at fighting extortion activities per se, but also to minimize the negative effects such activities have on the economic development in a region.

Although the question of economic growth under extortion is a highly relevant real world problem, only little work has been done to rigorously analyze which factors determine the effect of extortion activities in a region. Recent quantitative work on gang-related crime has rather focused on the problem of optimal crime fighting as such (e.g. Baveja et al. (1997), Dawid and Feichtinger (1996), Levitt (1997)) than on the implications of crime given optimal counter-strategies. Extortion activities in particular have been recently analyzed by Konrad and Skaperdas (1998) using game theoretic methods. They analyze the strategic interaction of gangs, shopowners and the police and characterize the optimal strategies of gangs and police in a static model. However, in the second part of their analysis they assume that the decisions of shop-owners about whether to enter the area

\footnotetext{
${ }^{1}$ See Jankowski (1991), Gambetta and Reuter (1995) or for example Los Angeles Times (1998a, 1998b) for reports on the impact of gang activities in the greater Los Angeles area on local businesses.

${ }^{2}$ To mention one of many examples, economic activities in San Francisco Chinatown have sharply decreased since the late 1970s due to local extortion activities (see Los Angeles Times (2000)).

${ }^{3}$ See also Anders (1997).
} 
or not depend on the relation of expected sales returns to expected extortion payments. Using this model they argue that in the case of a forward looking gang, which takes into account the effect of their action on the number of entrants, the efforts of both sides (gang and police) in equilibrium are smaller than if the gang is myopic and ignores this effect. Konrad and Skaperdas (1998) also observe that shop-owners in gang affected areas are reluctant to use expensive equipment and thus the capital invested in local businesses is comparably small, however they do not explicitly include such effects into their model. The goal of this paper is to use a dynamic game theoretical model to explicitly address the question of how extortion affects the development of local businesses. As an indicator of the development of local businesses we use overall capital which has been invested by local shopowners. We will not only examine how different time preferences of gangs and police influence this development but, also consider the effect of the size of private labour demand, the attitude of the local inhabitants towards criminal behavior, police corruption and the interplay of these parameters. The goal is to characterize certain combinations of environmental parameters which makes a region particularly vulnerable to economic decay in the presence of organized crime and to derive policy implications for regional planners.

The paper is organized as follows. We present the model in section 2, derive a Markov perfect equilibrium of the game in section 3 and characterize the parameter constellations which lead to growth or stagnation in section 4 . We conclude with a discussion of our findings and some policy implications in section 5 .

\section{The Model}

In order to investigate the effect extortion activities of criminal gangs have on the economic growth in an area we consider a model of dynamic strategic interaction between a representative shopkeeper, a gang, and the police. A differential game model is used to describe this dynamic interaction and the solution concept we use is that of a Markov perfect equilibrium (see e.g. Basar and Olsder, 1995). The differential game formulation should be seen as the limit case of a model with discrete time steps where the length of the time interval between the steps, $\Delta$, tends to 0 . Whereas such a differential game approach makes some restrictive assumptions about the information players use compared to analyzing the full extensive form game (players only react to the current value of the state variable) it has 
been established as a very useful approach to examine problems of capital accumulation (see Fudenberg and Tirole (1991), chap. 13). Our focus here is on the understanding of the development of the shop-owners' capital stock, and thus a differential game approach is a good compromise which on the one hand covers the crucial aspects of the problem and on the other hand allows a very precise characterization of the dynamic properties of the system in equilibrium. There are issues, like for example the credibility of the gangs' threats, which could only be addressed using a full extensive form game ${ }^{4}$, however these questions are not in the scope of this analysis.

Consider a shop-owner who at time $t$ owns a capital stock of $K(t)$ which is completely invested in his shop. The shop-owner immediately re-invests all net profits of operating his shop and without any interference of criminal gangs this would lead to a growth rate of capital of $r>0$. To keep the model as simple as possible we assume a constant growth rate. Using a decreasing growth rate would make an analytical solution of the model much harder but should not change the qualitative results concerning growth and stagnation $^{5}$. At any time $t$ the gang asks the shopkeeper for an amount $X_{\Delta}(t)$ and threatens to destroy the shop if the sum is not paid. The shopkeeper can either accept or reject this deal. In case he rejects, it is assumed that the shop-owner will leave the area at the end of the subsequent time interval. However, his shop is burned down within the subsequent time interval with probability $F\left(\Delta ; e_{g}, e_{p}\right)$ where $e_{g}(t)$ is the effort of the gang to keep up the threat and $e_{p}(t)$ is the effort of the police force to protect the shopkeeper. This probability could depend on the number of shops police has to protect, but since the evolution of the number of shops in a region is not determined endogeneously in our model, for reasons of simplicity we assume a constant number of shops. Also, we assume that investment in the shop does not change the size of the shop in a way which makes it harder to protect for a police force of given size. The shopkeeper is assumed to be only concerned

\footnotetext{
${ }^{4}$ see Konrad and Skaperdas (1997)

${ }^{5}$ By assuming that the shop-owner re-invests all profits in his shop we ignore the possibility of using outside investment options. Whereas this is clearly a simplification, the introduction of an outside option with constant interest rate $v$ would not alter the structure of our results on stagnation respectively growth in the long run. As long as shop-owners always choose the currently more attractive option the crucial condition for long run growth would just be that the net growth of capital in equilibrium is larger than $v$ rather than larger than 0 . If it is assumed that shop-owners allocate their profits between their shop and the outside option in an intertemporally optimal way they have to take strategic effects into account and a three player differential game would result. We assert that such considerations are beyond the typical small shop-owner and hence model their behavior as being myopic.
} 
about his expected capital stock at the end of the period and thus pays the demanded sum only if the expected damage in $[t, t+\Delta]$ resulting from rejection is larger or equal than the amount extorted (we do not explicitly take into account transaction costs of the shop-owner for transferring his capital out of the area, but formally they can be included in $F$ ). We assume that the gang knows this and therefore at any time exactly extorts the amount which makes the shopkeeper indifferent between accepting and rejecting the offer. Thus, the gang demands

$$
X_{\Delta}=F\left(\Delta ; e_{g}, e_{p}\right) e^{r \Delta} K(t) .
$$

This leads to a capital stock at $t+\Delta$ of:

$$
K(t+\Delta)=e^{r \Delta} K(t)\left(1-F\left(\Delta ; e_{g}, e_{p}\right)\right) .
$$

For $\Delta \rightarrow 0$ we get the state dynamics

$$
\dot{K}=\left(r-f\left(e_{g}, e_{p}\right)\right) K,
$$

where $f\left(e_{g}, e_{p}\right)=\lim _{\Delta \rightarrow 0} \frac{F\left(\Delta ; e_{g}, e_{p}\right)}{\Delta}$. We call $f\left(e_{g}, e_{p}\right)$ the threat rate.

The game has now been reduced to a two player game between the gang and the police. The gang maximizes the discounted future income stream from its extortion activities, which is at any time $t$ given by the difference between the amount extorted and the costs of the effort which is invested to keep up the threat. The gang's objective function is therefore given by

$$
\begin{aligned}
\max _{e_{g}} & \int_{0}^{\infty} e^{-\rho_{g} t}\left(X-c_{g}\left(e_{g}\right)\right) d t \\
= & \int_{0}^{\infty} e^{-\rho_{g} t}\left(K f\left(e_{g}, e_{p}\right)-c_{g}\left(e_{g}\right)\right) d t
\end{aligned}
$$

Police can by investing costly effort protect the shop from the gang and reduce the probability that the gang can carry out its threat. Due to public pressure and monitoring police has to invest a minimal effort $\gamma \geq 0$, but additional effort $e_{p}$ is at the discretion of the local police force. An un-corrupted police force tries to facilitate economic growth in the area by maximizing the amount invested by the shop-owners. However, it is well known that corruption of parts of the police force by local gangs is a serious problem in many gang infected areas. If police is corrupted it will participate in the gangs' profits and will rather try to keep these profits large. We include this possibility in our model by introducing a corruption parameter $\kappa$ which describes the degree to which the criminal gang was able 
to corrupt the police force. Police tries to maximize a weighted sum of the shop-owners' and the gangs' income, where the weight of the shop-owners income is $1-\kappa$ and the weight of the gangs income is $\kappa$. Thus, the objective function of the police is

$$
\begin{gathered}
\max _{e_{p}} \quad \int_{0}^{\infty} e^{-\rho_{p} t}\left[\kappa\left(X-c_{g}\left(e_{g}\right)\right)+(1-\kappa)(r K-X)-c_{p}\left(e_{p}\right)\right] d t \\
=\quad \int_{0}^{\infty} e^{-\rho_{p} t}\left[\kappa\left(f\left(e_{g}, e_{p}\right) K-c_{g}\left(e_{g}\right)\right)+(1-\kappa)\left(\left(r-f\left(e_{g}, e_{p}\right)\right) K-c_{p}\left(e_{p}\right)\right)\right] d t
\end{gathered}
$$

Both cost functions satisfy

$$
c_{i}(0)=0, c_{i}^{\prime}>0, c_{i}^{\prime \prime}>0 .
$$

It is further assumed that $r<\min \left[\rho_{g}, \rho_{p}\right]$ which guarantees that the objective functions of both players are always finite. The structure of the differential game between the gang and the police derived here is somehow related to resource exploitation games (see e.g. Feichtinger and Dockner (1994)). The shop-owners capital stock is the resource which is exploited by the gang and protected by the police force (at least if it is not corrupt). As usual in these types of dynamic games (but also in the static analysis of Konrad and Skaperdas (1998)) the evolution of the number of offenders (i.e. gang members) and number of potential victims (i.e. shops) is ignored and

\section{A Markov Perfect Equilibrium}

We will characterize a Markov perfect equilibrium solution for the game described above. Both players choose their level of effort depending only on the current capital stock of the shop-owners, i.e. $e_{g}(t)=e_{g}(K(t)), e_{p}(t)=$ $e_{p}(K(t))$. Define $S$ as the set of all measurable functions from $[0, \infty) \rightarrow$ $[0, \infty)$ such that $(1)$ has a well defined unique solution for all $K_{0} \in[0, \infty)$. A Markov perfect equilibrium is given by a pair of strategies $e_{g}^{*} \in S, e_{p}^{*} \in S$ such that

$$
\begin{gathered}
e_{g}^{*} \in \operatorname{argmax}_{e_{g} \in S} \int_{0}^{\infty} e^{-\rho_{g} t}\left(K f\left(e_{g}(K), e_{p}^{*}(K)\right)-c_{g}\left(e_{g}(K)\right)\right) d t \\
e_{p}^{*} \in \operatorname{argmax}_{e_{p} \in S} \int_{0}^{\infty} e^{-\rho_{p} t}\left[\kappa\left(f\left(e_{g}^{*}(K), e_{p}(K)\right) K-c_{g}\left(e_{g}^{*}(K)\right)\right)\right. \\
\left.+(1-\kappa)\left(r-f\left(e_{g}^{*}(K), e_{p}(K)\right)\right) K-c_{p}\left(e_{p}(K)\right)\right] d t
\end{gathered}
$$

for any $K_{0} \in[0, \infty)$, where the state dynamics is given by (1). It is well known that Markov perfect equilibria satisfy the condition of strong time 
consistency and thus do not involve any incredible threats on any side (Basar and Olsder (1995)).

In what follows we assume that both cost functions and the threat rate have Cobb-Douglas form:

$$
\begin{aligned}
f\left(e_{g}, e_{p}\right) & =\Phi e_{g}^{\alpha_{g}}\left(\gamma+e_{p}\right)^{\alpha_{p}} \\
c_{g}\left(e_{g}\right) & =c_{g} e_{g}^{\beta_{g}} \\
c_{p}\left(e_{p}\right) & =c_{p}\left(\gamma+e_{p}\right)^{\beta_{p}},
\end{aligned}
$$

where $\alpha_{g}>0, \alpha_{p}<0, \beta_{i}>1, i \in\{g, p\}$. Also, we assume that $\beta_{i}>\left|\alpha_{i}\right| i \in$ $\{g, p\}$ which corresponds to decreasing returns of money spent on extortion respectively crime control. The parameter $\Phi$ expresses the attitude of the local population towards the gang (in how far do witnesses of gang activities interfere with them, how cooperative are they in police investigations, etc.). Finding a Markov perfect equilibrium of a differential game is a challenging task since general solution methods exist only for certain special classes of games like linear quadratic games (see e.g. Mehlmann (1988)). This holds not only true for analytic solutions but also for numerical ones ${ }^{6}$. Although the game developed above belongs to none of the standard classes we can nevertheless characterize a Markov perfect solution for the 'symmetric' case where the efficiency of a dollar spent by both players decreases at the same rate, i.e. where a doubling of expenses of both sides leaves the threat rate unaffected:

Proposition 1 Assume $\frac{\alpha_{g}}{\beta_{g}}=\frac{\left|\alpha_{p}\right|}{\beta_{p}}:=\alpha$. Then for sufficiently small values of $\kappa$ in $[0,1]$ there exist constants $\lambda_{g} \in(0,1)$ and $\lambda_{p} \in(-1, \infty)$ such that the feedback strategy pair

$$
\begin{aligned}
& e_{g}^{*}(K)=\left[\alpha \Phi \frac{\left(1-\lambda_{g}\right)^{1+\alpha} c_{p}^{\alpha}}{\left(1+\lambda_{p}-2 \kappa\right)^{\alpha} c_{g}^{1+\alpha}}\right]^{\frac{1}{\beta_{g}}} K^{\frac{1}{\beta_{g}}} \\
& e_{p}^{*}(K)=\left[\alpha \Phi \frac{\left(1-\lambda_{g}\right)^{\alpha}\left(1+\lambda_{p}-2 \kappa\right)^{1-\alpha}}{c_{p}^{1-\alpha} c_{g}^{\alpha}}\right]^{\frac{1}{\beta_{p}}} K^{\frac{1}{\beta_{p}}}-\gamma
\end{aligned}
$$

constitutes a Markov perfect equilibrium of the game for sufficient small $\gamma$.

\footnotetext{
${ }^{6}$ Unfortunately, up to now it is not quite clear how for example iteration methods based on the contraction theorem, or shooting methods which can be used to numerically solve dynamic optimization problems, can be applied in such a differential game framework.
} 
Proof: The Hamiltonians for the two players read:

$$
\begin{aligned}
& H_{g}\left(K, e_{g}, e_{p}, \lambda_{g}\right)=\left(1-\lambda_{g}\right) K f\left(e_{g}, e_{p}\right)-c_{g}\left(e_{g}\right)+\lambda_{g} r K \\
& H_{p}\left(K, e_{g}, e_{p}, \lambda_{p}\right)=\left(1-\kappa+\lambda_{p}\right) r K-\left(1-2 \kappa+\lambda_{p}\right) K f\left(e_{g}, e_{p}\right)-\kappa c_{g}\left(e_{g}\right)-c_{p}\left(e_{p}\right),
\end{aligned}
$$

where $\lambda_{g}, \lambda_{p}$ denote the shadow prices of the shop-owners capital stock for both players. To show that (5) and (6) indeed constitute a Markov perfect equilibrium we make the assumption that in this equilibrium the (current value) value functions of both players are linear in $K$ (note that obviously both value functions have to be zero for $K=0$ ):

$$
V_{g}(K)=\lambda_{g} K, \quad V_{p}(K)=\lambda_{p} K .
$$

We first show that under this assumption the optimal policies of the two players are given by (5) and (6). Afterwards, we establish that $V_{g}$ and $V_{p}$ are indeed the value functions by showing that they satisfy the Hamilton Jacobi Bellman equation and the transversality conditions.

Noting that the coefficients of $K$ in the value functions are identical to the shadow prices we get the following first order condition for the gang:

$$
\begin{aligned}
& \frac{\partial H_{g}}{\partial e_{g}} \\
& \quad=\left(1-\lambda_{g}\right) K f_{1}\left(e_{g}, e_{p}\right)-c_{g}^{\prime}\left(e_{g}\right) \\
& =\alpha_{g}\left(1-\lambda_{g}\right) K \frac{f\left(e_{g}, e_{p}\right)}{e_{g}}-\beta_{g} \frac{c_{g}\left(e_{g}\right)}{e_{g}} \\
& =0 .
\end{aligned}
$$

Thus,

$$
c_{g}\left(e_{g}^{*}\right)=\alpha\left(1-\lambda_{g}\right) K f\left(e_{g}^{*}, e_{p}^{*}\right)
$$

On the other hand,

$$
\begin{aligned}
& \frac{\partial H_{p}}{\partial e_{p}} \\
& \quad=-\left(1+\lambda_{p}-2 \kappa\right) K f_{2}\left(e_{g}, e_{p}\right)-c_{p}^{\prime}\left(e_{p}\right) \\
& =-\alpha_{p}\left(1+\lambda_{p}-2 \kappa\right) K \frac{f\left(e_{g}, e_{p}\right)}{\gamma+e_{p}}-\beta_{p} \frac{c_{p}\left(e_{p}\right)}{\gamma+e_{p}} \\
& =0
\end{aligned}
$$

which gives

$$
c_{p}\left(e_{p}^{*}\right)=\alpha\left(1+\lambda_{p}-2 \kappa\right) K f\left(e_{g}^{*}, e_{p}^{*}\right) .
$$


Thus, the threat rate is in equilibrium constant in $K$ :

$$
\begin{aligned}
f\left(e_{g}^{*}, e_{p}^{*}\right) & =\Phi\left(\frac{c_{g}\left(e_{g}^{*}\right)}{c_{g}}\right)^{\frac{\alpha_{g}}{\beta_{g}}}\left(\frac{c_{p}\left(e_{p}^{*}\right)}{c_{p}}\right)^{\frac{\alpha_{p}}{\beta_{p}}} \\
& =\Phi\left(\frac{\left(1-\lambda_{g}\right) c_{p}}{\left(1+\lambda_{p}-2 \kappa\right) c_{g}}\right)^{\alpha} .
\end{aligned}
$$

Taking into account that both cost functions are strictly increasing in the corresponding effort and $\alpha<1$ this also implies that the points satisfying the first order conditions are global maxima and simple algebra establishes that they are given by (5) and (6).

To finish the proof we have to show that there exist values of $\lambda_{g}$ and $\lambda_{p}$ such that the value functions $V_{i}(K)$ satisfy the transversality conditions

$$
\lim _{t \rightarrow \infty} e^{-\rho_{g} t} V_{g}(K)=\lim _{t \rightarrow \infty} e^{-\rho_{p} t} V_{p}(K)=0
$$

for all equilibrium trajectories of $K$ and the Hamilton-Jacobi-Bellman equations (see e.g. Shimomura, 1991)

$$
\rho_{i} V_{i}(K)=H_{i}\left(K, e_{g}^{*}, e_{p}^{*}, V_{i}^{\prime}\right) \quad i \in\{g, p\} .
$$

The transversality conditions hold due to our assumption that $r<$ $\min \left(\rho_{g}, \rho_{p}\right)$. Comparing the coefficients of $K$ on the left and right hand side of both Hamilton Jacobi Bellman equations gives after some simplification the following conditions:

$$
\begin{aligned}
\left(\rho_{g}-r\right) \lambda_{g}= & \Phi(1-\alpha) \frac{\left(1-\lambda_{g}\right)^{1+\alpha} c_{p}^{\alpha}}{\left(1+\lambda_{p}-2 \kappa\right)^{\alpha} c_{g}^{\alpha}} \\
\left(\rho_{p}-r\right) \lambda_{p}= & (1-\kappa) r-\Phi(1+\alpha) \frac{\left(1-\lambda_{g}\right)^{\alpha} c_{p}^{\alpha}\left(1+\lambda_{p}-2 \kappa\right)^{1-\alpha}}{c_{g}^{\alpha}} \\
& -\kappa \Phi \alpha \frac{\left(1-\lambda_{g}\right)^{1+\alpha} c_{p}^{\alpha}}{\left(1+\lambda_{p}-2 \kappa\right)^{\alpha} c_{g}^{\alpha}} .
\end{aligned}
$$

Thus, we have to show that there exist constants $\lambda_{g}$ and $\lambda_{p}$ which satisfy (9) and (10). We show this for $\kappa=0$ which by continuity implies that linear value functions also exist for sufficiently small positive values of $\kappa$. Considering (9) we realize that as long as $\lambda_{p}>-1$ the left hand side is smaller than the right hand side for $\lambda_{g}=0$, whereas the inequality is reversed for $\lambda_{g}=1$. Since the left hand side is increasing and the right hand side decreasing in $\lambda_{g}$ there has to be unique point $\lambda_{g}^{*}\left(\lambda_{p}\right)$ where the left hand side equals the 
right hand side. It is further easy to see that $\lambda_{g}^{*}\left(\lambda_{p}\right)$ is a decreasing function. We insert this function $\lambda_{g}^{*}\left(\lambda_{p}\right)$ for $\lambda_{g}$ in the expression on the right hand side of (10) and realize that the resulting expression is a decreasing function in $\lambda_{p}$ for $\kappa=0$. Obviously, the left hand side of (10) increases with $\lambda_{p}$. Furthermore, the left hand side is negative for $\lambda_{p}=-1$ whereas the right hand side is larger or equal zero. Together with the observation that for $\lambda_{p} \rightarrow \infty$ the left hand side goes to plus infinity and the right hand side to minus infinity this shows that there is a unique pair $\left(\lambda_{g}, \lambda_{p}\right) \in(0,1) \times(-1, \infty)$ which satisfies (10) and (9).

Of course, even for small levels of corruption, the feedback equilibrium described here is not necessarily the unique feedback solution of the game (see e.g. Feichtinger and Wirl, 1993), however we have shown that it is the only one where the shadow prices of a unit increase of the shop-owners capital stock is constant for both players. We will be mainly interested in the case of small $\kappa$ where we have a unique equilibrium with constant shadow prices, but nevertheless like to point out that for larger values of $\kappa$ we might lose this uniqueness property and even the existence of such an equilibrium all together. This is made precise in the following Corollary where we also exactify what we mean by 'small $\kappa$ ':

Corollary 1 Assume $\gamma=0$. For

$$
\kappa \leq \kappa_{1}:=\frac{\rho_{p}(1-\alpha)}{2 \rho_{p}(1-\alpha)+\alpha \rho_{g}-r}
$$

there exist unique values $\lambda_{g} \in(0,1)$ and $\lambda_{p} \in(-1, \infty)$ such that (5) and (6) constitute a Markov perfect equilibrium. Further, there exists a constant

$$
\kappa_{2} \in\left(\kappa_{1}, \kappa_{3}\right),
$$

where

$$
\kappa_{3}:=\frac{\rho_{p}}{2 \rho_{p}-r}
$$

such that there exist two equilibria of the form $(5,6)$ for $\kappa \in\left(\kappa_{1}, \kappa_{2}\right)$ a unique equilibrium for $\kappa=\kappa_{2}$, and no equilibrium with equilibrium strategies (5) and (6) for $\kappa>\kappa_{2}$. For $\kappa \in\left(\kappa_{1}, \kappa_{2}\right]$ the branch of equilibria with the lower value of $f\left(e_{g}^{*}, e_{p}^{*}\right)$, is the continuous extension of the line of unique equilibria for $\kappa \leq \kappa_{1}$. The unique equilibria for $\kappa \leq \kappa_{1}$ and the equilibria with lower threat rate for $\kappa \in\left(\kappa_{1}, \kappa 2\right]$ are called regular equilibria, whereas the branch of 
equilibria with higher $f\left(e_{g}^{*}, e_{p}^{*}\right)$ for $\kappa \in\left(\kappa_{1}, \kappa_{2}\right)$ are called collusive extinction equilibria. For regular equilibria the equilibrium threat rate increases with $\kappa$, whereas for collusive extinction equilibria the threat rate decreases with $\kappa$ and goes to infinity as $\kappa$ approaches $\kappa_{1}$ from above.

Proof: See Appendix.

We sketch the correspondence between the level of corruption and equilibrium threat rates in figure 1.

Insert figure 1 here!

The implications of this Corollary are quite interesting. As long as the level of corruption is below $\kappa_{2}$ there exists a regular equilibrium where police objectives are basically opposite to that of the gang and the equilibrium threat rate slowly and continuously increases with the level of corruption. If we consider a scenario where corruption slowly increases from below $\kappa_{1}$ to above $\kappa_{1}$ we should definitely expect to stay on this equilibrium branch also for $\kappa>\kappa_{1}$. However, for $\kappa$ slightly larger than $\kappa_{1}$ there suddenly appears a second equilibrium with an enormously high threat rate. To interpret the behavior in this additional equilibrium branch, we conclude from the arguments given in the proof of the corollary that for $\kappa$ only slightly larger than $\kappa_{1}$ the shadow price of $K$ for the gang is close to $\lambda_{g}=1$ and that of the police close to $\lambda_{p}=2 \kappa-1$, which implies that both efforts are almost zero. However, although they are both very small the gang effort is by a huge factor larger than that of the police inducing a large threat rate. Hence, in this equilibrium the police completely colludes with the gang, refuses to protect shopowners at all, which allows the gang to extort more or less the complete current capital stock of the shopowners at low costs. Of course this implies a fast breakdown of economic activities in the region. Although this gives a nice interpretation of the second equilibrium we do not consider such kind of behavior realistic, at least in developed countries, and will in the future analysis concentrate only on the branch of regular equilibria. Note that the collusive extinction equilibrium is only admissible if the minimal effort of police $\gamma$ is extremely low. It should also be pointed out, that the level of corruption needed for the existence of the collusive extinction equilibrium is higher the more farsighted the gang is. Furthermore, this level increases with increasing $\rho_{p}$ if $\alpha \rho_{g}>r$ and decreases otherwise.

Finally, we like to point out that the arguments used in the proof of Corollary 1 imply that in the regular equilibrium the value function of the 
gang always increases with the level of police corruption, whereas it is impossible to make a general statement about how the value function of the police depends on $\kappa$. In the collusive extinction equilibrium the value function of the gang would decrease in $\kappa$, and, since this seems to be quite counterintuitive, this is another argument for focusing on the regular equilibrium.

\section{Characterization of Growth and Stagnation Sce- narios}

In what follows we will concentrate on the qualitative properties of the regular equilibrium derived in the previous section. First, we would like to point out that whereas in equilibrium the shadow value of the shop-owner's capital for the gang is always positive, it might be negative for the police force. The intuitive explanation is that, whereas additional capital always increases the amount a gangs can extort, for the police an increase in capital stock - depending on future efforts on both sides - might lead to an increase of future losses due to an increase in extorted funds. In such a case the local police force sees a large capital stock of the shop-owners as a burden since it realizes that it will be costly to defend this capital stock in the future and prevent future losses of the shop-owner due to extortion. It is intuitively clear and can also be easily derived from (9) and (10) that the absolute value of both shadow values decreases with the size of the corresponding discount rate. Considering the equilibrium levels of effort we therefore conclude that the more myopic the gang is (the larger $\rho_{g}$ is) the higher the effort levels on both sides are. The effect of an increase of $\rho_{p}$ on the equilibrium effort level depends on whether police considers $K$ as an asset or a burden. In the first case increasing myopia of the police leads to decreasing police efforts but to increasing efforts of the gang, whereas both effects are reversed if $\lambda_{p}<0$. Both levels of effort are smaller the more hostile the local population is towards extortion activities but increase with the current level of capital stock. In equilibrium police always counters large efforts by the gang with a high own effort level.

Focusing on the threat rate, we observe that the danger of a shopkeeper to lose his enterprise, if he does not pay the gang, is in equilibrium independent of the current capital stock. As already observed, a higher capital stock leads to larger efforts both from the gang and police and we can see that the net effect on the threat rate is zero. Since $f\left(e_{g}^{*}, e_{p}^{*}\right)$ is independent of $K$ the capital stock in equilibrium either continuously grows $\left(r>f\left(e_{g}^{*}, e_{p}^{*}\right)\right)$ or - for $r<f\left(e_{g}^{*}, e_{p}^{*}\right)$ - monotonously decreases towards zero. 
In the first of the two scenarios, police can handle the problem of extortion so well that economic growth in the area is only slowed down but not prevented by the gang's activities. We call this the reduced growth case. In the second scenario however criminal activity is such a large obstacle for business owners that the capital stock in the area shrinks continuously and in the long run all shops will vanish. We call this the stagnation case. To understand the impact gang activities will have on an area it is important to examine under which conditions these two qualitatively completely different scenarios occur. Unfortunately, we cannot explicitly express the equilibrium threat rate $f\left(e_{g}^{*}, e_{p}^{*}\right)$ as a function of the model parameters (we can not analytically solve for $\lambda_{g}$ and $\lambda_{p}$ ). In the following proposition we are however able to characterize under which parameter constellations the two cases occur. The introduction of the following notation simplifies the expressions in the following analysis considerably and also provides a better intuition of the results. We denote by $\tilde{\rho}_{g}=\frac{\rho_{g}}{r}, \tilde{\rho}_{p}=\frac{\rho_{p}}{r}$ the ratio of the two players discount rate to the capital growth rate $\left(\tilde{\rho}_{i}>1, i \in\{g, p\}\right)$ and by $\tilde{\Phi}=\frac{\Phi}{r}\left(\frac{c_{p}}{c_{g}}\right)^{\alpha}$ the ratio of threat rate and growth rate if gang and police spend identical amounts of money. This ratio is determined by the attitude of the local inhabitants towards the gang's activities and we say that an area is gang hostile if $\tilde{\Phi}<1$ whereas it is gang friendly if $\tilde{\Phi}>1$. Using this notation we can derive the following characterization of areas where growth can be upheld in spite of extortion activities.

Proposition 2 If the two players behave according to the regular Markov perfect equilibrium characterized in Proposition 1 and Corollary 1, then the shop-owners capital stock continually grows if and only if

$$
\kappa<\frac{\tilde{\rho}_{p}\left(\tilde{\rho}_{g}-\alpha\right)-\tilde{\Phi}^{\frac{1}{\alpha}}\left(\tilde{\rho}_{g}-1\right)\left(\tilde{\rho}_{p}+\alpha\right)}{\tilde{\rho}_{g}-\alpha+\alpha\left(\tilde{\rho}_{g}-1\right)+2\left(\tilde{\rho}_{g}-\alpha\right)\left(\tilde{\rho}_{p}-1\right)} .
$$

Proof: First, we characterize for which parameter constellation the equilibrium capital stock neither grows nor shrinks. This holds whenever $f\left(e_{g}^{*}, e_{p}^{*}\right)=$ $r$. In the proof of Corollary 1 we have derived that $\frac{f\left(e_{g}^{*}, e_{p}^{*}\right)}{r}=x$ holds if and only if (26) holds. Inserting $x=1$ into (26) and solving for $\kappa$ gives

$$
\kappa=\frac{\tilde{\rho}_{p}\left(\tilde{\rho}_{g}-\alpha\right)-\tilde{\Phi}^{\frac{1}{\alpha}}\left(\tilde{\rho}_{g}-1\right)\left(\tilde{\rho}_{p}+\alpha\right)}{\tilde{\rho}_{g}-\alpha+\alpha\left(\tilde{\rho}_{g}-1\right)+2\left(\tilde{\rho}_{g}-\alpha\right)\left(\tilde{\rho}_{p}-1\right)} .
$$

We know from Corollary 1 that the threat rate in the regular equilibrium increases with $\kappa$ and this proves our claim. 
Using this proposition we may now analyze what role different characteristics of the area under consideration, of the local gangs and of the local police force play in the economic development of a gang infected region. We will state our observations as corollaries and discuss each of them in some detail. We formulate the results for cases where the police force has not been corrupted by the gang with the understanding that these statements are also true for small $\kappa>0$.

Corollary 2 If police is not corrupt $(\kappa=0)$ sufficiently small values of $\alpha$ lead to growth if the area is gang hostile but to stagnation if the area is gang friendly.

Proof: For $\kappa=0$ the condition for growth is

$$
\tilde{\rho}_{p}\left(\tilde{\rho}_{g}-\alpha\right)>\tilde{\Phi}^{\frac{1}{\alpha}}\left(\tilde{\rho}_{g}-1\right)\left(\tilde{\rho}_{p}+\alpha\right) .
$$

For $\tilde{\Phi}>1$ the right hand side goes to infinity for $\alpha \rightarrow 0$, for $\tilde{\Phi}<1$ it goes to zero. Since the left hand side is positive but finite the corollary follows.

Remember that the parameter $\alpha$ expresses how fast the effect of one dollar spent by the gang respectively the police decreases with increasing expenses. A small $\alpha$ corresponds to quickly decreasing returns. If it is assumed that costs on both sides are mainly due to expenses for additional labour, a small $\alpha$ can be interpreted as an expression of a rather wage-inelastic labour supply faced by gang and police. Thus we like to interpret large values of $\alpha$ as corresponding to a case of significant unemployment in the region, whereas small $\alpha$ stands for a strong demand for labour in the private sector ${ }^{7}$. Corollary 2 shows that a competitive private labour demand helps a region to handle extortion only if the environment is gang hostile. If the environment is gang friendly a strong private labour market will rather lead to a faster decay of a gang infected area. We would like to make two observations on this rather surprising point. It has to be realized that our results hold under the assumption that both sides act optimally. The problem in many regions with a low (legal) private labor demand might be that police forces are in general not as flexible to adapt their labour force and efforts as they should

\footnotetext{
${ }^{7}$ We assume here that the local shop-owners demand for labour is insignificant, otherwise $\beta_{i}$ would have to depend on $K$.
} 
be $^{8}$. Thus, in many cases the gangs are better able to exploit a large $\alpha$ and the equilibrium observation that a weak labor market can actually help a region to overcome crime might not hold true in real world situations.

Now let us turn to the questions how the discount rate of the gang and police influence the prospects of growth in a region.

Corollary 3 In the presence of a non-corrupt police force growth in an area is sustained if the discount rate of the criminal gang is sufficiently small. On the other hand, if the environment is gang friendly extortion leads to stagnation if the discount rate of the gang is sufficiently large.

Proof: Keeping in mind that we always assume $\rho_{i}>r$ we realize that $\tilde{\rho}_{g}>1$. For $\tilde{\rho}_{g} \rightarrow 1$ the right hand side of (15) goes to zero and the first part of the Corollary follows. On the other hand, the coefficient of $\tilde{\rho}_{g}$ on the right hand side of $(15)$ is $\tilde{\Phi}^{\frac{1}{\alpha}}\left(\tilde{\rho}_{p}+\alpha\right)$ which for $\tilde{\Phi}>1$ is larger than $\tilde{\rho}_{p}$, the coefficient of $\tilde{\rho}_{g}$ on the left hand side. Accordingly the inequality (15) is violated for large values of $\tilde{\rho}_{g}$.

According to this corollary a gang does the more harm to the economic development of a region the more myopic it is. This is not only in accordance with the results Konrad and Skaperdas (1998) derive in their static framework but also with observable facts. Short lived gangs in regions of turmoil like Russia have a much more severe effect on the economic development of their neighborhood than established criminal organizations with a longer planning horizon like the Italian Mafia. Even if the environment is gang hostile a large discount rate of the gang might lead to stagnation, especially if the police is rather far-sighted and labour supply is inelastic. If an area should keep growing even in the presence of a myopic criminal gang the expression $\tilde{\Phi}^{\frac{1}{\alpha}}\left(1+\frac{\alpha}{\tilde{\rho}_{p}}\right)$ has to be smaller than 1 . This means that the area has to be gang hostile $(\tilde{\Phi}<1)$, prospects on the private labour market should be good ( $\alpha$ small) and the police force should act myopically ( $\tilde{\rho}_{p}$ large). Thus, contrary to the gang, police does not necessarily facilitate long term growth when acting far-sighted.

Corollary 4 Assume that police is not corrupt. Whenever, equilibrium behavior leads to persistent growth for some discount rate $\tilde{\rho}_{p}^{*}$, the shop-owners

\footnotetext{
${ }^{8}$ Of course there are several factors reducing the flexibility of police recruitment. Among others, screening and training of candidates, existing long term labour contracts, etc.
} 
capital stock also grows for any larger discount rate $\tilde{\rho}_{p} \geq \tilde{\rho}_{p}^{*}$. If

$$
\tilde{\Phi}<\left(\frac{\tilde{\rho}_{g}-\alpha}{\tilde{\rho}_{g}-1}\right)^{\alpha}
$$

a sufficiently large discount rate of the police leads to persistent capital growth. On the other hand, if (16) is violated stagnation results for all values $\tilde{\rho}_{p} \geq 1$.

Proof: We rewrite condition (15) as

$$
\tilde{\rho}_{p}\left(\tilde{\rho}_{g}-\alpha-\tilde{\Phi}^{\frac{1}{\alpha}}\left(\tilde{\rho}_{g}-1\right)\right)>\tilde{\Phi}^{\frac{1}{\alpha}} \alpha\left(\tilde{\rho}_{g}-1\right) .
$$

If this inequality holds for $\tilde{\rho}_{p}=1$ we have

$$
\tilde{\rho}_{g}-\alpha>\tilde{\Phi}^{\frac{1}{\alpha}}\left(\tilde{\rho}_{g}-1\right)(1+\alpha)
$$

and therefore the coefficient of $\tilde{\rho}_{p}$ in the inequality above is positive and the inequality holds for all $\tilde{\rho}_{p}>1$. If the inequality does not hold for $\tilde{\rho}_{p}=1$ but for some larger value $\tilde{\rho} *_{p}>1$ the coefficient of $\tilde{\rho}_{p}$ has to be positive and thus the inequality has to hold for all $\tilde{\rho}_{p} \geq \tilde{\rho}_{p}^{*}$. The second part of the corollary is trivial since (16) guarantees that the coefficient of $\tilde{\rho}_{p}$ is positive. Finally, if (16) is violated, we know on one hand that the coefficient of $\tilde{\rho}_{p}$ is negative and, on the other hand, that the inequality is violated for $\tilde{\rho}_{p}=1$. Hence, it is violated for all $\tilde{\rho}_{p} \geq 1$ and we always get stagnation.

Thus, we have the very interesting result that myopic behavior of the police force never leads to stagnation if more far-sighted behavior leads to growth. The reverse is not true and heavy discounting on the side of the police force might yield persistent growth in situation where more far-sighted behavior would have implied stagnation. The discount rate of the local police force may depend on the evaluation criteria used (what time intervals are considered in reviews), the flexibility of the labour contracts, or the personnel turnover in the local police force. According to our results keeping the police force flexible with considerable personnel turnaround in the units and providing incentives based on short term goals and successes is important to prevent the economic decay of a region. Intuitively, the reason, why a frequent exchange of police officers is advantageous, is that a 'no hope' scenario is avoided where officers fear that they will have to fight against 
the gang forever without much hope for final success. This might lead to indifferent low-effort behavior and our analysis shows that this might be even optimal. Exchanging officers regularly and rewarding them based on short run successes is a good way to deal with this problem. In the framework of our model this corresponds to a large discount rate for the police force.

Finally, let us examine how harmful corruption of the police force is under the different scenarios. A first obvious observation is that a large level of corruption increases the equilibrium threat rate and the danger of stagnation. We can however also obtain some insights about which environmental conditions aggravate the effect of a (partly) corrupt police force. To this end, let us again consider the cases where non-corrupt behavior leads to growth in the region. We have seen above that the capital stock in the region keeps growing in the presence of extortion if either the environment is gang hostile and $\alpha$ is small and/or $\tilde{\rho}_{g}$ is close to one. In the limit case $\left(\alpha \rightarrow 0\right.$ and $\left.\tilde{\rho}_{g} \rightarrow 1\right)$ the crucial level of corruption which distinguishes the scenarios of growth and stagnation is given by $\kappa=\frac{\tilde{\rho}_{p}}{2 \tilde{\rho}_{p}-1}$. Hence, we see that, for small $\alpha$ and $\tilde{\rho}_{p}{ }^{9}$ the crucial level of corruption decreases with increasing $\tilde{\rho}_{p}$. Hence, we realize that myopia of the police has two opposite effects. It facilitates growth as long as the police force is not corrupt, but on the other side the danger to growth generated by corruption is larger in such a case. Whereas this insight might question our findings above that frequent exchange of officers has positive impact on the development of a region, it might also be argued that corruption is more likely to occur if officers stay in the same area for a long time. Such effects however are not included in our model and we abstain from a thorough discussion.

\section{Conclusions}

In this paper we have used a dynamic game theoretical model to analyze the impact different characteristics of a region have on the effect of extortion activities on the local economic development. Using the solution concept of a Markov perfect equilibrium we have identified conditions for continued growth of the capital stock of local shop-owners and for stagnation. The main policy implications for regional planers who want to ensure continued growth are the following: (i) If the local population in an area is not supportive of gang activities and private sector demand for labor is large the capital stock of local shop owners keeps growing in spite of the extor-

\footnotetext{
${ }^{9}$ Note that in the extreme case $\alpha=0, \tilde{\rho}_{g}=1$ the crucial level of corruption converges to $\kappa_{3}$ and therefore all scenarios where the equilibrium exists lead to growth.
} 
tion activities of the gang. (ii) If there exists excess supply of labor in a gang infected area it is essential for the police force to expand and accept additional current costs. Such behavior prevents stagnation of economic development in the region and is optimal in the long run. (iii) It is essential to guarantee that the police force heavily discounts future utility. Myopic behavior of the police makes stagnation in general less likely but on the other hand aggravates the effect of possible corruption of parts of the police force by the gangs. Furthermore, we have also shown that myopia of the local gangs has negative effects on the economic development of a region. These qualitative findings and the resulting policy implications are all backed by a formal dynamic analysis but also allow a nice intuitive understanding of the inter-temporal effects at work. If the model is calibrated using data from a certain region the size of these different effects could be quantified and more concrete policy implications could be derived. Thus, the presented analysis could be the starting point for highly interesting empirical analyses.

The model we have used is in many aspects very stylized and several extensions come to mind. In the current model the attitude of the local population towards gang activities is externally given and constant over time. However, in real world scenarios it is evident that this attitude depends heavily on previous gang activities and changes over time. Thus, the parameter $\Phi$ in our model should be endogenized. Furthermore, the structure of the market might be influenced by the gang's activities, since a strong presence of the gang might diminish competition in this area ${ }^{10}$. Taking this into account the parameter $r$ in our model would depend on the gang's effort. Finally, here we treated the degree of corruption of the police force as given, whereas it might be seen as the result of costly effort of the gang. Endogenizing any of these aspects would make an interesting extension of the present model and bring it closer to describing the complex real world relationships between different economic activities, criminal activities and phenomena like corruption. It seems to us, however, that such extensions would preclude an analytical solution of the resulting game and numerical methods would have to be used.

\footnotetext{
${ }^{10}$ We are grateful to a referee for pointing out this effect.
} 


\section{References}

Anders, A. (1997), 'Observations on the Development of Small Private Enterprises in Russia', Post-Soviet Geography and Economics, 38, 191 -205.

Basar, T. and Olsder, G.J. (1995), Dynamic Noncooperative Game Theory, Academic Press, London, England.

Baveja, A., Caulkins, J.P., Liu, W., Batta, R. and Karwan, M.H. (1997), 'When Haste Makes Sense: Cracking Down on Street Markets for Illicit Drugs', Socio-Economic Planning Sciences, 31, 293 - 306.

Dawid, H. and Feichtinger, G. (1996), 'Optimal Allocation of Drug Control Efforts: A Differential Game Analysis', Journal of Optimization Theory and Applications, 91, 2, 279 - 297.

The Economist (1994), 'Crime in Russia: the High Price of Freeing Markets', 19, February.

Feichtinger, G. and Dockner, E.J. (1994), 'Environmentalists versus Resources Exploiters: A Dynamic Game Analysis' in V.G.L. Paredes (Ed.), International Symposium on Systems Analysis and Management Decisions in Forestry, Universidad Austral of Chile Press, Valdivia, Chile, pp. 392398.

Feichtinger, G. and Wirl, F. (1993), 'A Dynamic Variant of the Battle of the Sexes', International Journal of Game Theory, 22, 359-380.

Fiorentini, G. and Peltzman, S. (Eds.) (1995), The Economics of Organized Crime, Cambridge University Press, Cambridge, MA.

Fudenberg, D. and Tirole, J. (1991), Game Theory, MIT Press.

Gambetta, D. and Reuter, P. (1995), 'Conspiracy Among the Many: the Mafia in Legitimate Industries', in Fiorentini and Peltzman (1995).

Jankowski, M. (1991), Islands in the Street: Gangs and American Urban Society, University of California Press, Berkeley.

Konrad, K. and Skaperdas, S. (1997), 'Credible Threats in Extortion', Journal of Economic Behavior and Organization, 33, 23 - 39.

Konrad, K. and Skaperdas, S. (1998), 'Extortion', Economica, 65, 461 - 477.

Levitt, S.D. (1997), 'Using Electoral Cycles in Police Hiring to Estimate the Effect of Police on Crime', American Economic Review, 87, 270 - 290.

Los Angeles Times (1998a), 'Risky Business for Immigrants', 01/18/98, B-1. 
Los Angeles Times (1998b), 'City Attorney Seeks Injunction Against Street Gang Members', 08/02/98, B-3.

Los Angeles Times (2000), 'New Year, New Attitude Toward Chinatown Extortion', 02/14/00, A-3.

Mehlmann, A. (1988), Applied Differential Games, Plenum Press, New York.

Shimomura, K. (1991), The Feedback Equilibria of a Differential Game of Capitalism, Journal of Economic Dynamics and Control, 15, 317-338. 


\section{Appendix}

\section{Proof of Corollary 1:}

The threat rate is given by

$$
f\left(e_{g}^{*}, e_{p}^{*}\right)=\Phi\left(\frac{\left(1-\lambda_{g}\right) c_{p}}{\left(1+\lambda_{p}-2 \kappa\right) c_{g}}\right)^{\alpha}
$$

where $\lambda_{g}$ and $\lambda_{p}$ are solutions of (9) and (10). Let us define the parameters

$$
\tilde{\rho}_{g}=\frac{\rho_{g}}{r}, \tilde{\rho}_{p}=\frac{\rho_{p}}{r}, \tilde{\Phi}=\frac{\Phi}{r}\left(\frac{c_{p}}{c_{g}}\right)^{\alpha}
$$

The above equations can then be written as

$$
x=\frac{f\left(e_{g}^{*}, e_{p}^{*}\right)}{r}=\tilde{\Phi}\left(\frac{1-\lambda_{g}}{1+\lambda_{p}-2 \kappa}\right)^{\alpha}
$$

where $\lambda_{g}$ and $\lambda_{p}$ are solutions of

$$
\begin{gathered}
\left(\tilde{\rho}_{g}-1\right) \lambda_{g}=\tilde{\Phi}(1-\alpha) \frac{\left(1-\lambda_{g}\right)^{1+\alpha}}{\left(1+\lambda_{p}-2 \kappa\right)^{\alpha}} \\
\left(\tilde{\rho}_{p}-1\right) \lambda_{p}=(1-\kappa)-\tilde{\Phi}(1+\alpha) \frac{\left(1-\lambda_{g}\right)^{\alpha}}{\left(1+\lambda_{p}-2 \kappa\right)^{\alpha-1}}-\kappa \tilde{\Phi} \alpha \frac{\left(1-\lambda_{g}\right)^{1+\alpha}}{\left(1+\lambda_{p}-2 \kappa\right)^{\alpha}}
\end{gathered}
$$

To find an expression of the threat rate or equivalently of $x$ as function of the model parameters only, we eliminate $\lambda_{g}$ and $\lambda_{p}$ from equation (19) making use of (20) and (21).

Substituting (19) into (20) gives

$$
\left(\tilde{\rho}_{g}-1\right) \lambda_{g}=(1-\alpha)\left(1-\lambda_{g}\right) x \Rightarrow \lambda_{g}=\frac{x(1-\alpha)}{\tilde{\rho}_{g}-1+x(1-\alpha)}
$$

Substituting (19) into (21) gives

$$
\left(\tilde{\rho}_{p}-1\right) \lambda_{p}=(1-\kappa)-(1+\alpha)\left(1+\lambda_{p}-2 \kappa\right) x-\kappa \alpha\left(1-\lambda_{g}\right) x
$$

Solving (23) for $\lambda_{p}$ gives

$$
\lambda_{p}=\frac{1-\kappa-(1+\alpha)(1-2 \kappa) x-\kappa \alpha\left(1-\lambda_{g}\right) x}{\tilde{\rho}_{p}-1+x(1+\alpha)}
$$


Equation (19) is equivalent to

$$
\tilde{\Phi}^{\frac{1}{\alpha}}\left(1-\lambda_{g}\right)-x^{\frac{1}{\alpha}}\left(1+\lambda_{p}-2 \kappa\right)=0
$$

Substituting (22) and (24) into (25) we obtain the following implicit equation for $x$ :

$$
\begin{aligned}
& \frac{\tilde{\phi}^{\frac{1}{\alpha}}\left(1-\tilde{\rho}_{g}\right)\left[1-\tilde{\rho}_{p}-x(1+\alpha)\right]-x \frac{1+\alpha}{\alpha}\left[\tilde{\rho}_{p}(1-\alpha)(1-2 \kappa)+\kappa\left(1-\alpha \tilde{\rho}_{g}\right)\right]}{\left[1-\tilde{\rho}_{g}-x(1-\alpha)\right]\left[1-\tilde{\rho}_{p}-x(1+\alpha)\right]} \\
&+\frac{x \frac{1}{\alpha}\left(1-\tilde{\rho}_{g}\right)\left[\tilde{\rho}_{p}(1-2 \kappa)+\kappa\right]}{\left[1-\tilde{\rho}_{g}-x(1-\alpha)\right]\left[1-\tilde{\rho}_{p}-x(1+\alpha)\right]}=0
\end{aligned}
$$

Taking only the numerator of $(26) f\left(e_{g}^{*}, e_{p}^{*}\right) / r$ is solution of the equation $g(x)=0$, where the function $g(x)$ is of the form

$$
g(x)=-a x^{1+\frac{1}{\alpha}}-b x^{\frac{1}{\alpha}}+c x+d
$$

with

$$
\begin{aligned}
a & =\tilde{\rho}_{p}(1-\alpha)-\kappa\left[2 \tilde{\rho}_{p}(1-\alpha)+\alpha \tilde{\rho}_{g}-1\right] \\
b & =\left(\tilde{\rho}_{g}-1\right)\left[\tilde{\rho}_{p}-\kappa\left(2 \tilde{\rho}_{p}-1\right)\right] \\
c & =\tilde{\phi}^{\frac{1}{\alpha}}\left(\tilde{\rho}_{g}-1\right)(1+\alpha) \\
d & =\tilde{\phi}^{\frac{1}{\alpha}}\left(\tilde{\rho}_{g}-1\right)\left(\tilde{\rho}_{p}-1\right)
\end{aligned}
$$

Note that both $a$ and $b$ are linear in $\kappa$ and due to

$$
2 \tilde{\rho}_{p}(1-\alpha)+\alpha \tilde{\rho}_{g}-1>2 \tilde{\rho}_{p}(1-\alpha)+\alpha-1=(1-\alpha)\left(2 \tilde{\rho}_{p}-1\right)>0
$$

we can conclude that $a$ and $b$ are decreasing in $\kappa$. Furthermore, $\mathrm{b}$ is negative iff $\kappa>\frac{\tilde{\rho}_{p}}{2 \tilde{\rho}_{p}-1}$. For $\kappa=\frac{\tilde{\rho}_{p}}{2 \tilde{\rho}_{p}-1}$ the coefficient $a$ is given by

$$
a=\frac{\alpha \tilde{\rho}_{p}\left(1-\tilde{\rho}_{g}\right)}{2 \tilde{\rho}_{p}-1}<0
$$

and since $a$ is decreasing in $\kappa$ we get that $b<0$ implies $a<0$. We define $\kappa_{1}$ as the unique positive value where $a=0$ and $\kappa_{3}>\kappa_{1}$ as the unique positive value where $b=0$. It is easy to see that these values are given by (11) and (13).

We have $g(0)>0, g^{\prime}(0)>0$,

$$
g^{\prime \prime}(x)=x^{\frac{1}{\alpha}-2}\left(-\frac{a}{\alpha}\left(1+\frac{1}{\alpha}\right) x-\frac{b}{\alpha}\left(\frac{1}{\alpha}-1\right)\right),
$$


and

$$
\lim _{x \rightarrow \infty} g(x)= \begin{cases}-\infty & \kappa \leq \kappa_{1} \\ \infty & \kappa>\kappa_{1}\end{cases}
$$

Thus, we have a unique positive root of $g(x)$ for $\kappa \leq \kappa_{1}$. On the other hand, for $\kappa$ slightly larger than $\kappa_{1}$ we have two positive roots of $g(x)$ (note that there cannot be more than two since $g^{\prime \prime}$ changes sign only once) and for $\kappa \rightarrow \kappa_{1}+$ the smaller of the two converges towards the unique root for $\kappa=\kappa_{1}$, whereas the larger goes to infinity. Furthermore, for an arbitrary given $x, g(x)$ increases with increasing $\kappa$ which implies that the smaller of the two positive roots wanders to the right, whereas the larger of the two roots wanders to the left for increasing $\kappa$. They collide for $\kappa=\kappa_{2}$ and vanish as $\kappa$ is further increased. Note that $\kappa_{2}<\kappa_{3}$ has to hold since $g(x)>0$ holds for all $x \geq 0$ and $\kappa \geq \kappa_{3}$. 


\section{Figure Captions}

Figure 1: The equilibrium threat rate for $\rho_{g}=\rho_{p}=1, r=0.3, \Phi=$ $0.2, \alpha=0.8$ and $\kappa \in[0,0.35]$. For this parameter constellation we have $\kappa_{1}=0 . \dot{2}, \kappa_{2}=0.3202$ and $\kappa_{3}=0.588$. 


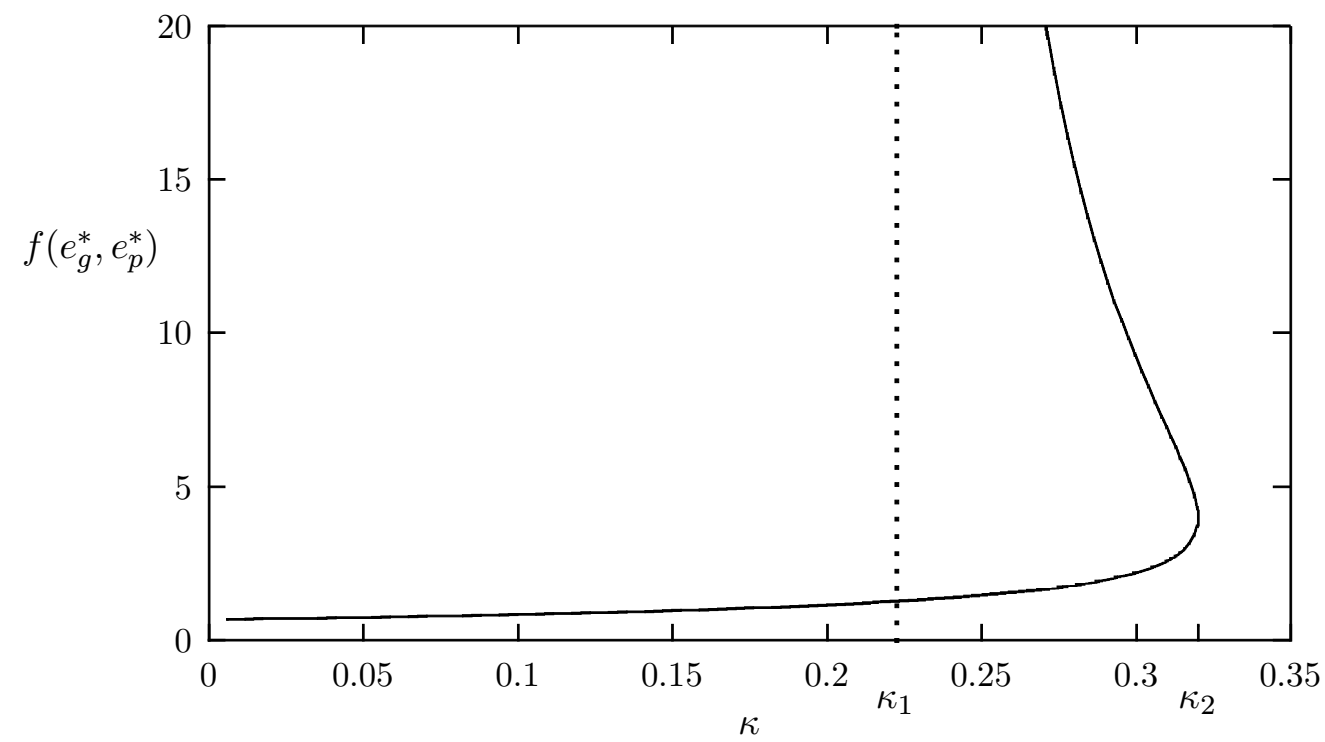

Figure 1 